\title{
A Low-Complexity Eigenfilter Design Method for Channel Shortening Equalizers for DMT Systems
}

\author{
Andre Tkacenko, Student Member, IEEE, and P. P. Vaidyanathan, Fellow, IEEE
}

\begin{abstract}
We present a new low-complexity method for the design of channel shortening equalizers for discrete multitone modulation systems using the eigenfilter approach. In contrast to other such methods which require a Cholesky decomposition for each delay parameter value used, ours requires only one such decomposition. Simulation results show that our method performs nearly optimally in terms of observed bit rate.
\end{abstract}

Index Terms-Channel-shortening equalizers, eigenfilter, fractionally spaced equalizers.

\section{INTRODUCTION}

D UE TO THE recent interest in discrete multitone (DMT) modulation systems, the design of time-domain equalizers (TEQs) or channel-shortening equalizers has received much attention [2], [3], [6]. Because of the long impulse response of typical channels encountered in DMT systems, such as twisted pair telephone lines [7], TEQs are required to shorten the overall channel response to one sample more than the length of the cyclic prefix used.

Several methods proposed for the design of TEQs involve a minimum mean-squared error (MMSE) design of the effective channel (namely, the cascade of the channel and equalizer) [1], [2] and not the equalizer directly. With such methods, the equalizer is then obtained using the well-known orthogonality principle. Other methods, such as the maximum shortening signal-to-noise ratio (MSSNR) method of [5] and the minimum intersymbol interference (min-ISI) method of [3], deal directly with the equalizer coefficients and are examples of eigenfilter methods [10]. Though these methods have been shown to perform well, they require a different Cholesky decomposition for each delay parameter value used.

In contrast, a new eigenfilter method recently introduced requires only one such decomposition [6]. The goal of this method is to minimize the delay spread of the effective channel. Though this method was shown to be less prone to synchronization errors than others, it does not account for the cyclic prefix length or any knowledge of noise statistics. Due to noise source models for DMT systems, such as near-end crosstalk (NEXT) and far-end crosstalk (FEXT) [7], it is only natural to exploit such knowledge to obtain a better equalizer.

In this letter, we generalize the eigenfilter method of [6] in many respects. First, we apply it to the single-input mul-

Paper approved by N. Al-Dhahir, the Editor for Space-Time, OFDM, and Equalization of the IEEE Communications Society. Manuscript received May 6, 2002; revised August 6, 2002 and January 8, 2003. This work was supported in part by the Office of Naval Research under Grant N00014-99-1-1002.

The authors are with the Department of Electrical Engineering, California Institute of Technology, Pasadena, CA 91125 USA (e-mail: ppvnath@systems.caltech.edu).

Digital Object Identifier 10.1109/TCOMM.2003.814235

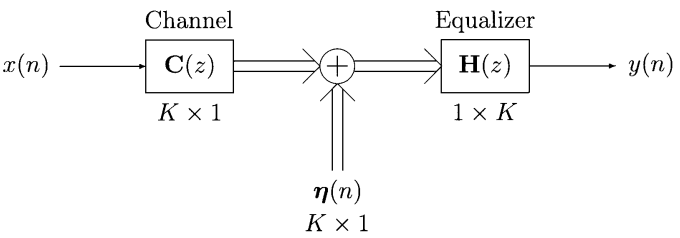

Fig. 1. SIMO-MISO channel/equalizer model.

tiple-output (SIMO) channel and multiple-input single-output (MISO) equalizer model, which can be used for the design of fractionally spaced equalizers (FSEs) as shown in [8]. Secondly, we incorporate the cyclic prefix length and effects due to noise. Simulation results provided show that our method performs comparably with other methods in terms of achievable bit rate with less computational complexity. Parts of this letter have been presented by the authors in recent conference proceedings [8], [9].

\section{Proposed Eigenfilter Design Method}

Consider the SIMO-MISO channel/equalizer model of Fig. 1. Here, $K$ is the oversampling factor for FSEs. We make the following assumptions here.

- The channel $\mathbf{C}(z)$ is a known finite impulse response (FIR) filter of length $L_{c}$.

- The equalizer $\mathbf{H}(z)$ is a FIR filter of length $L_{e}$.

- The input $x(n)$ is zero-mean and white with variance $\sigma_{x}^{2}$.

- The noise $\boldsymbol{\eta}(n)$ is a zero-mean wide-sense stationary (WSS) random process with autocorrelation $\mathbf{R}_{\boldsymbol{\eta} \boldsymbol{\eta}}(k)$.

- The processes $x(n)$ and $\boldsymbol{\eta}(n)$ are uncorrelated.

Note that the output $y(n)$ can be expressed as $y(n)=x_{f}(n)+$ $q(n)$, where $x_{f}(n)$ and $q(n)$ are, respectively, the filtered signal and noise processes given by

$$
x_{f}(n)=c_{\mathrm{eff}}(n) * x(n), \quad q(n)=\mathbf{h}(n) * \boldsymbol{\eta}(n)
$$

and $c_{\mathrm{eff}}(n)$ is the effective channel given by $c_{\mathrm{eff}}(n) \triangleq \mathbf{h}(n) *$ c $(n)$. We want the equalizer to shorten the effective channel $c_{\text {eff }}(n)$ and minimize the noise power $\sigma_{q}^{2}$ with respect to the signal power $\sigma_{x_{f}}^{2}$. To that end, we choose $\mathbf{h}(n)$ to minimize the objective function

$$
J \triangleq \alpha J_{\text {short }}+(1-\alpha) J_{\text {noise }}, \quad 0 \leq \alpha \leq 1
$$

where $J_{\text {short }}$ and $J_{\text {noise }}$ are defined as follows:

$$
\begin{gathered}
J_{\text {short }} \triangleq \frac{\sum_{n} f(n-\Delta)\left|c_{\text {eff }}(n)\right|^{2}}{\sum_{n}\left|c_{\text {eff }}(n)\right|^{2}} \\
J_{\text {noise }} \triangleq \frac{\sigma_{q}^{2}}{\sigma_{x_{f}}^{2}}=\frac{\sigma_{q}^{2}}{\sigma_{x}^{2} \sum_{n}\left|c_{\text {eff }}(n)\right|^{2}} .
\end{gathered}
$$


Here, $J_{\text {short }}$ is a channel shortening objective, $J_{\text {noise }}$ is the noise-to-signal ratio, and $\alpha$ is a tradeoff parameter between these two objectives. Also, $\Delta$ is the delay of the shortened channel, and $f(n)$ is a "penalty" function, which is any nonnegative function used to penalize different values of $c_{\text {eff }}(n)$. The special case where $K=1, \alpha=1$, and $f(n)=n^{2}$ corresponds to the objective analyzed in [6].

Though $\alpha$ is arbitrary, we have heuristically opted to use

$$
\alpha=\alpha_{0} \triangleq \frac{P_{\mathrm{ISI}}}{P_{\mathrm{ISI}}+P_{\text {noise }}} .
$$

Here, $P_{\text {ISI }}$ is the intersymbol interference (ISI) power present in $\mathbf{c}(n)$ before equalization, which is $\sigma_{x}^{2}$ times the difference of the energy of $\mathbf{c}(n)$ and the energy of the $\left(L_{\mathrm{CP}}+1\right)$ length window of $\mathbf{c}(n)$ with maximum energy, where $L_{\mathrm{CP}}$ is the cyclic prefix length. Also, $P_{\text {noise }}$ is the input noise power, namely, $\operatorname{Tr}\left[\mathbf{R}_{\boldsymbol{\eta} \boldsymbol{\eta}}(0)\right]$. Finding the optimal choice of $\alpha$ for a given criterion is still an open problem. However, for maximizing bit rate, it was found (see Section IV) that $\alpha_{0}$ in (3) yielded good results.

To further incorporate the cyclic prefix length in the design, we have chosen the penalty function

$$
f(n)=f_{\mathrm{CP}}(n) \triangleq \begin{cases}0, & 0 \leq n \leq L_{\mathrm{CP}} \\ 1, & \text { otherwise }\end{cases}
$$

Note that $f_{\mathrm{CP}}(n-\Delta)$ penalizes uniformly any samples of $c_{\text {eff }}(n)$ outside of $n \in\left[\Delta, \Delta+L_{\mathrm{CP}}\right]$.

\section{AnAlysis of the OBJective Function $J$}

Let us define the quantities shown in the equation at the bottom of the page. These quantities have the following sizes:

- $\mathbf{h}: 1 \times K L_{e}$;

- $\mathbf{C}: K L_{e} \times\left(L_{c}+L_{e}-1\right)$;

- $\boldsymbol{\Lambda}:\left(L_{c}+L_{e}-1\right) \times\left(L_{c}+L_{e}-1\right)$;

- $\mathbf{R}_{\boldsymbol{\eta}}: K L_{e} \times K L_{e}$.

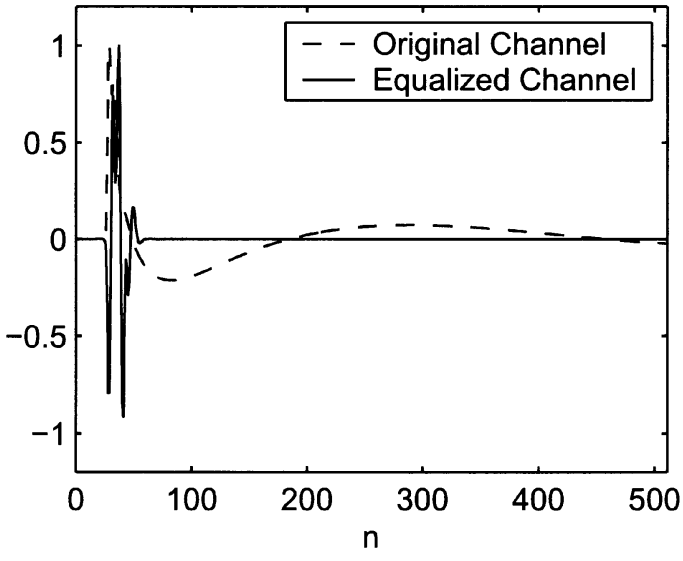

Fig. 2. Original and equalized channel impulse responses for CSA loop \#1.

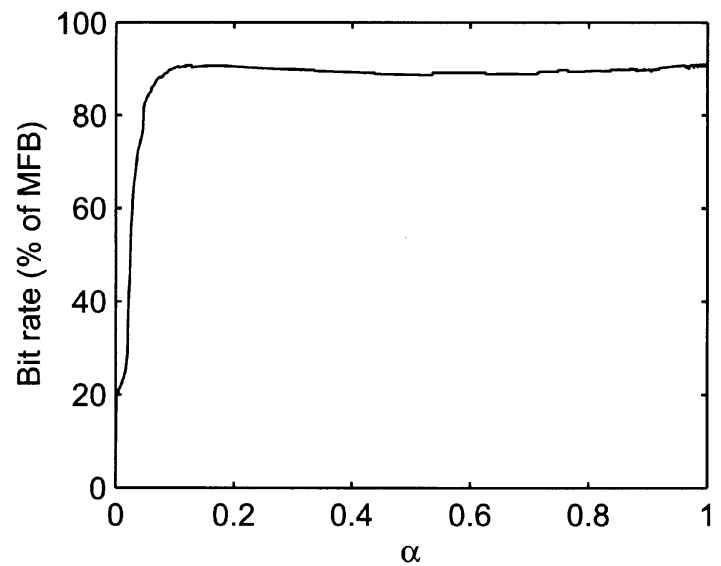

Fig. 3. Observed bit rate (as a percentage of the MFB bit rate) as a function of the tradeoff parameter $\alpha$ for CSA loop \#1.

Then, from (2), we can show that we have

$$
J_{\text {short }}=\frac{\mathbf{h} \mathbf{C} \boldsymbol{\Lambda} \mathbf{C}^{\dagger} \mathbf{h}^{\dagger}}{\mathbf{h} \mathbf{C} \mathbf{C}^{\dagger} \mathbf{h}^{\dagger}}, \quad J_{\text {noise }}=\frac{\mathbf{h} \mathbf{R}_{\boldsymbol{\eta}} \mathbf{h}^{\dagger}}{\sigma_{x}^{2} \mathbf{h} \mathbf{C} \mathbf{C}^{\dagger} \mathbf{h}^{\dagger}} .
$$

Assuming $\mathbf{C}$ has a full rank of $K L_{e}$, then $\mathbf{A} \triangleq \mathbf{C C}^{\dagger}$ is positive definite. As such, it has a Cholesky decomposition of the form

$$
\begin{aligned}
& \mathbf{h} \triangleq\left[\begin{array}{llll}
\mathbf{h}(0) & \mathbf{h}(1) & \cdots & \mathbf{h}\left(L_{e}-1\right)
\end{array}\right] \\
& \mathbf{C} \triangleq\left[\begin{array}{ccccccc}
\mathbf{c}(0) & \mathbf{c}(1) & \cdots & \mathbf{c}\left(L_{c}-1\right) & \mathbf{0} & \cdots & \mathbf{0} \\
\mathbf{0} & \mathbf{c}(0) & \mathbf{c}(1) & \cdots & \mathbf{c}\left(L_{c}-1\right) & \ddots & \vdots \\
\vdots & \ddots & \ddots & \ddots & & \ddots & \mathbf{0} \\
\mathbf{0} & \cdots & \mathbf{0} & \mathbf{c}(0) & \mathbf{c}(1) & \cdots & \mathbf{c}\left(L_{c}-1\right)
\end{array}\right] \\
& \boldsymbol{\Lambda} \triangleq \operatorname{diag}\left(f(0-\Delta), f(1-\Delta), \ldots, f\left(\left(L_{c}+L_{e}-2\right)-\Delta\right)\right) \\
& \mathbf{R}_{\boldsymbol{\eta}} \triangleq\left[\begin{array}{cccc}
\mathbf{R}_{\boldsymbol{\eta} \boldsymbol{\eta}}(0) & \mathbf{R}_{\boldsymbol{\eta} \boldsymbol{\eta}}(1) & \cdots & \mathbf{R}_{\boldsymbol{\eta} \boldsymbol{\eta}}\left(L_{e}-1\right) \\
\mathbf{R}_{\boldsymbol{\eta} \boldsymbol{\eta}}(-1) & \mathbf{R}_{\boldsymbol{\eta} \boldsymbol{\eta}}(0) & \ddots & \vdots \\
\vdots & \ddots & \ddots & \mathbf{R}_{\boldsymbol{\eta} \boldsymbol{\eta}}(1) \\
\mathbf{R}_{\boldsymbol{\eta} \boldsymbol{\eta}}\left(-\left(L_{e}-1\right)\right) & \cdots & \mathbf{R}_{\boldsymbol{\eta} \boldsymbol{\eta}}(-1) & \mathbf{R}_{\boldsymbol{\eta} \boldsymbol{\eta}}(0)
\end{array}\right]
\end{aligned}
$$


TABLE I

ObServed Bit Rates for CSA Loops \#1-8 Using Various TEQ Design Methods. (Bit Rates EXPRessed as Percentage of MFB MAXIMUM ACHIEVABLE BIT RATE FOR EACH LOOP.)

\begin{tabular}{c|c|c|c|c|c|c|c|c}
\hline \multirow{2}{*}{$\begin{array}{c}\text { CSA } \\
\text { loop \# }\end{array}$} & \multicolumn{7}{|c|}{ Observed bit rate as $a \%$ of the MFB maximum bit rate } & $\begin{array}{c}\text { MFB } \\
\text { maximum } \\
\text { achievable } \\
\text { bit rate }\end{array}$ \\
\cline { 2 - 9 } & $\begin{array}{c}\text { MMSE } \\
\text {-UTC [1] }\end{array}$ & $\begin{array}{c}\text { MMSE } \\
\text {-UEC }[1]\end{array}$ & $\begin{array}{c}\text { GSNR } \\
{[2]}\end{array}$ & $\begin{array}{c}\text { MSSNR } \\
{[5]}\end{array}$ & $\begin{array}{c}\text { min-ISI } \\
{[3]}\end{array}$ & $\begin{array}{c}\text { Delay Spread } \\
\text { Minimization [6] }\end{array}$ & $\begin{array}{c}\text { Proposed } \\
\text { Method }\end{array}$ & \\
\hline 1 & $82.4 \%$ & $83.3 \%$ & $84.8 \%$ & $92.6 \%$ & $95.0 \%$ & $77.4 \%$ & $91.1 \%$ & $2.844 \mathrm{Mbps}$ \\
\hline 2 & $81.1 \%$ & $86.6 \%$ & $89.3 \%$ & $86.4 \%$ & $95.3 \%$ & $66.8 \%$ & $91.1 \%$ & $3.068 \mathrm{Mbps}$ \\
\hline 3 & $80.3 \%$ & $81.1 \%$ & $83.1 \%$ & $90.4 \%$ & $95.3 \%$ & $80.2 \%$ & $92.3 \%$ & $2.643 \mathrm{Mbps}$ \\
\hline 4 & $62.7 \%$ & $74.9 \%$ & $74.2 \%$ & $88.6 \%$ & $93.2 \%$ & $51.9 \%$ & $92.9 \%$ & $1.933 \mathrm{Mbps}$ \\
\hline 5 & $79.7 \%$ & $72.6 \%$ & $75.2 \%$ & $81.9 \%$ & $93.7 \%$ & $68.5 \%$ & $89.5 \%$ & $2.839 \mathrm{Mbps}$ \\
\hline 6 & $77.8 \%$ & $80.7 \%$ & $80.6 \%$ & $89.5 \%$ & $94.3 \%$ & $80.6 \%$ & $92.8 \%$ & $2.350 \mathrm{Mbps}$ \\
\hline 7 & $64.1 \%$ & $78.2 \%$ & $79.6 \%$ & $90.3 \%$ & $93.9 \%$ & $72.2 \%$ & $89.8 \%$ & $2.519 \mathrm{Mbps}$ \\
\hline 8 & $69.1 \%$ & $78.1 \%$ & $80.7 \%$ & $88.6 \%$ & $91.8 \%$ & $79.0 \%$ & $90.6 \%$ & $2.325 \mathrm{Mbps}$ \\
\hline
\end{tabular}

$\mathbf{A}=\mathbf{G}^{\dagger} \mathbf{G}$, where $\mathbf{G}$ is a nonsingular $K L_{e} \times K L_{e}$ matrix. Defining the $K L_{e} \times 1$ column vector $\mathbf{v}$ as $\mathbf{v} \triangleq \mathbf{G h}^{\dagger}$, we have from (1) and (5)

$$
J=\frac{\mathbf{v}^{\dagger} \mathbf{T} \mathbf{v}}{\mathbf{v}^{\dagger} \mathbf{v}}
$$

$\mathbf{T} \triangleq \alpha\left[\left(\mathbf{G}^{-1}\right)^{\dagger} \mathbf{C} \boldsymbol{\Lambda} \mathbf{C}^{\dagger}\left(\mathbf{G}^{-1}\right)\right]+(1-\alpha)\left[\frac{1}{\sigma_{x}^{2}}\left(\mathbf{G}^{-1}\right)^{\dagger} \mathbf{R}_{\boldsymbol{\eta}}\left(\mathbf{G}^{-1}\right)\right]$.

As $\mathbf{T}$ is Hermitian, it follows by Rayleigh's principle that the minimum value of $J$ is $\lambda_{\min }$ where $\lambda_{\min }$ denotes the smallest eigenvalue of $\mathbf{T}$. Furthermore, $J=\lambda_{\min }$ iff $\mathbf{v}$ lies in the eigenspace corresponding to $\lambda_{\min }$. As $\mathbf{h}=\mathbf{v}^{\dagger}\left(\mathbf{G}^{-1}\right)^{\dagger}$, the optimum equalizer coefficients can be found from $\mathbf{v}$. One important point to note is that the Cholesky factor $\mathbf{G}$ does not depend on the delay parameter $\Delta$. Other eigenfilter methods, such as [3] and [5], have Cholesky factors that do depend on $\Delta$.

\section{Simulation ReSUlts}

Here we have opted to compare our proposed TEQ design method with others on the basis of observed bit rate. Data for the channel and noise was obtained from the Matlab DMTTEQ Toolbox [4]. We used the following typical asymmetric digital subscriber line (ADSL) input parameters:

- sampling frequency: $2.208 \mathrm{MHz}$, DFT size: 512, SNR gap: $9.8 \mathrm{~dB}$;

- $L_{c}=512, L_{\mathrm{CP}}=32, L_{e}=16, \sigma_{x}^{2}=21 \mathrm{dBm}$;

- NEXT noise model with eight disturbers [7] plus additive white Gaussian noise (AWGN) with power density -110 $\mathrm{dBm} / \mathrm{Hz}$.

Fig. 2 shows the original and equalized channel impulse responses for carrier service area (CSA) loop \#1 designed using our proposed method. Here, we chose $\alpha=\alpha_{0}$ as in (3) and $f(n)=f_{\mathrm{CP}}(n)$ as in (4). We varied the delay parameter $\Delta$ from 0 to 40 and chose the one which yielded the best bit rate. As we can see, our method shortened the channel quite well. In Fig. 3, we have plotted the observed bit rate (as a percentage of the matched-filter bound (MFB) maximum achievable bit rate [7]) as a function of the tradeoff parameter $\alpha$. Here, $\alpha_{0}=0.9977$ and yielded a percentage of 91.0819, whereas the optimum $\alpha$ was 0.998 with a percentage of 91.0852 . Clearly, the heuristic choice of $\alpha=\alpha_{0}$ from (3) yielded nearly optimal results as desired. From Fig. 3, we can see that performance remained relatively constant for $0.1 \leq \alpha \leq 1$, which heuristically means that for the simulation parameters chosen here, ISI is more of a problem than noise.

In Table I, we have tabulated the observed bit rates for CSA loops \#1-8 using the above parameters as a percentage of the MFB bit rate. The expression for the observed bit rate [3] takes into account both the ISI power of the effective channel as well as the effects due to the filtered noise. For each method considered except for the geometric SNR method (GSNR) [2], which requires nonlinear optimization, we varied the delay parameter $\Delta$ from 0 to 40 and chose the value that yielded the best bit rate. The optimum MMSE-unit energy constraint (UEC) method of [1] was used as the initial condition for the GSNR method. As was done in [3], the mean-squared error (MSE) parameter used was set to be $2 \mathrm{~dB}$ above the MSE obtained from the optimal MMSE-UEC equalizer. From Table I, we can see that our proposed method comes very close to the MFB maximum bit rate and is comparable with the min-ISI method of [3]. However, we should note that our proposed method requires less computational load, as we only require one Cholesky decomposition for all values of $\Delta$, as opposed to the min-ISI method which requires a different such decomposition for each $\Delta$. The MISO equalizers for FSEs designed using our method offer a further improvement over all the methods considered here (see [8] for more details), however, for sake of fair comparison and brevity, these results have been omitted.

\section{CONCLUDING REMARKS}

In this letter, we have generalized the delay spread minimization method of [6] to account for the cyclic prefix length as 
well as the noise encountered in the system. We showed that our method is less complex to implement than other common eigenfilter TEQ design methods, in that it only requires one Cholesky decomposition for all delay parameter values. From our simulation results, it was observed that our method came close to MFB maximum bit rate for all CSA loops considered, showing the merit of our proposed TEQ design method.

\section{REFERENCES}

[1] N. Al-Dhahir and J. M. Cioffi, "Efficiently computed reduced-parameter input-aided MMSE equalizers for ML detection: A unified approach," IEEE Trans. Inform. Theory, vol. 42, pp. 903-915, May 1996.

[2] — "A bandwidth-optimized reduced-complexity equalized multicarrier transceiver," IEEE Trans. Commun., vol. 45, pp. 948-956, Aug. 1997.
[3] G. Arslan, B. L. Evans, and S. Kiaei, "Equalization for discrete multitone transceivers to maximize bit rate," IEEE Trans. Signal Processing, vol. 49, pp. 3123-3135, Dec. 2001.

[4] Matlab DMTTEQ Toolbox, G. Arslan, B. Lu, and B. L. Evans. [Online]. Available: http://signal.ece.utexas.edu/ arslan/dmtteq/dmtteq.html

[5] P. J. W. Melsa, R. C. Younce, and C. E. Rohrs, "Impulse response shortening for discrete multitone transceivers," IEEE Trans. Commun., vol. 44, pp. 1662-1672, Dec. 1996.

[6] R. Schur and J. Speidel, "An efficient equalization method to minimize delay spread in OFDM/DMT systems," in Proc. IEEE ICC '01, vol. 1, Helsinki, Finland, June 2001, pp. 1-5.

[7] T. Starr, J. M. Cioffi, and P. J. Silverman, Understanding Digital Subscriber Line Technology. Upper Saddle River, NJ: Prentice-Hall, 1999.

[8] A. Tkacenko and P. P. Vaidyanathan, "Noise optimized eigenfilter design of time-domain equalizers for DMT systems," in Proc. IEEE ICC '02, vol. 1, New York, NY, Apr. 2002, pp. 54-58.

[9] - "Eigenfilter design of MIMO equalizers for channel shortening," in Proc. IEEE ICASSP '02, vol. 3, Orlando, FL, May 2002, pp. 2361-2364.

[10] P. P. Vaidyanathan and T. Q. Nguyen, "Eigenfilters: a new approach to least-squares FIR filter design and applications including Nyquist filters," IEEE Trans. Circuits Syst., vol. CAS-34, pp. 11-23, Jan. 1987. 